\title{
Complex 3D nanocoral like structures formed by copper nanoparticle aggregation on nanostructured zinc oxide rods
}

\author{
Robert Boyd, Fredrik Söderlind, Ulf Helmersson, Magnus Odén and Iris Pilch
}

Journal Article

\section{Tweet}

N.B.: When citing this work, cite the original article.

Original Publication:

Robert Boyd, Fredrik Söderlind, Ulf Helmersson, Magnus Odén and Iris Pilch, Complex 3D nanocoral like structures formed by copper nanoparticle aggregation on nanostructured zinc oxide rods, Materials letters (General ed.), 2016. 184(), pp.127-130.

http://dx.doi.org/10.1016/j.matlet.2016.08.023

Copyright: Elsevier

http://www.elsevier.com/

Postprint available at: Linköping University Electronic Press

http://urn.kb.se/resolve?urn=urn:nbn:se:liu:diva-132650

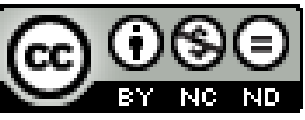




\title{
Complex 3D Nanocoral like Structures formed by Copper Nanoparticle
} Aggregation on Nanostructured Zinc Oxide Rods.

\author{
Robert D. Boyd ${ }^{a *}$, Fredrik Söderlind ${ }^{a}$, Ulf Helmersson ${ }^{a}$, Magnus Odén ${ }^{a}$ and Iris Pilch ${ }^{a}$ \\ a - IFM-Materials Physics, Linköping University, Linköping, 58183 Sweden.
}

\begin{abstract}
.
This paper reports a new strategy for nanoparticle surface assembly so that they form anisotropic fibril-like features, consisting of particles directly attached to each other, which can extend $500 \mathrm{~nm}$ from the surface. The particles are both formed and deposited in a single step process enabled via the use of a pulsed plasma based technique. Using this approach, we have successfully modified zinc oxide rods, up to several hundred nanometers in diameter, with $25 \mathrm{~nm}$ diameter copper nanoparticles for catalytic applications. The resulting structure could be modelled using a diffusion limited aggregation based approach. This gives the material the appearance of marine coral, hence the term nanocoral.
\end{abstract}

KEYWORDS: Pulsed Plasma, Nanostructured materials, scanning electron microscopy, focused ion beam (FIB), scanning transmission electron microscopy.

- - Corresponding author - robert.boyd@liu.se 


\section{Introduction}

One of the major challenges facing nanoscience is the assembly of nanoparticles into a specific architecture. Namely the ability to control, align and fix freely moving nanoparticles to a particular location and shape[1]. Controlling individual nanoparticles so they combine to form anisotropic constructs[2,3] has been proposed as a method to produce a range of nano-enabled materials. On the other hand, poor dispersion of copper nanoparticles can dramatically reduce their catalytic performance[4]. One of the most important applications for heterogeneous nanomaterials, due to their high surface-to-volume ratio, is in forming the basis for new and more effective catalysis. Copper $(\mathrm{Cu}) /$ zinc oxide $(\mathrm{ZnO})$ based nanomaterials are being extensively investigated as an effective catalysts for production of methanol from hydrogen and carbon dioxide, with many different preparation routes being proposed in order to optimise the $\mathrm{Cu} / \mathrm{ZnO}$ structure[5-8]. Here, $\mathrm{ZnO}$ rods are modified directly with copper nanoparticles using a pulsed plasma to sputter material from a hollow cathode with the aid of argon gas[9,10]. This is a direct method, producing and depositing the nanoparticles in a single step, without the need of any dispersing procedures or agents which could affect the properties, and therefore the catalytic efficiency, of the material. The resulting structure was determined using a combination of scanning electron microscopy (SEM), (scanning) transmission electron microscopy ((S)TEM) and focussed ion beam (FIB).

\section{Materials and Methods}

$\mathrm{ZnO}$ rods were produced by a previously reported method[11]. All nanoparticles were formed and deposited using a high power pulsed hollow cathode technique[9,10]. Gas (argon) is flowed through the hollow copper cathode so that a plasma discharge is formed between the cathode and an anode ring. Material is sputtered from the cathode by the plasma, which can then coalesce into particles once leaving the cathode, as it does so the particles gain a negative charge. The size of the particles is controlled by the plasma conditions (pressure, power and pulse frequency and duration) and also the spacing between the hollow cathode and anode ring[9]. The discharge parameters were kept constant at pressure $108 \mathrm{~Pa}$ (argon gas flow $60 \mathrm{sccm}$ ), frequency $700 \mathrm{~Hz}$, pulse width $50 \mu \mathrm{s}$, and peak current approx. 3A (average power of approx. 18W) and ran for 6 minutes The loading was changed by manipulating the deposition conditions for the nanoparticles. For this, the mesh enclosing the growth region was left at a floating potential or kept at a negative bias at $-20 \mathrm{~V}$. The negative bias results in a more focused deposition of nanoparticles because of their negative charge. In order to support a guided deposition of the nanoparticles onto the substrates, the substrates were positively biased at $10 \mathrm{~V}$. 
All SEM images and FIB cross section and TEM foil preparation were conducted using a 1540 EsB Cross Beam instrument (Zeiss, Germany). All SEM images were taken using a 5kV electron beam. Prior to FIB cross section preparation, a protective layer of platinum was deposited, first with the aid of the electron beam and subsequently the ion beam. Images of the FIB cross section were taken at an angle of $54^{\circ}$ with using an image tilt compensation of $36^{\circ}$. All (S)TEM analysis was taken using a Tecnai G2 TF 20 UT FEG TEM operated at $200 \mathrm{kV}$ (FEI, USA). High angle angular dark field (HAADF) and energy dispersive x-ray spectroscopy (EDS) combined with STEM analysis was taken using a camera length of $170 \mathrm{~mm}$. Specimens suitable for (S)TEM analysis were prepared by FIB milling and polishing[12].

\section{Results}

SEM images of various modified rods are shown in Figure 1 (additional images provided in supporting information). The samples were imaged both from above ( $0^{\circ}$ tilt $)$ and from the side $\left(54^{\circ}\right.$ tilt $)$ to give a closer representation of the true material structure. The rods are clearly visible with diameters between $50 \mathrm{~nm}$ to 400 $\mathrm{nm}$ and several micrometers in length. The top view appears to show individual or small clusters of particles deposited onto the rods, with fewer particles attached to a sample with a high loading of particles (Figure 1(a), mesh with a negative potential) compared to a lower loading case (Figure 1(b), mesh with a floating potential). Imaging from the side, however, reveals a different structure with the particles aggregating onto the rods to form fibril or tendril type structures concentrated at the top of the rods. Smaller aggregates and individual nanoparticles are seen on the sides of the rods.
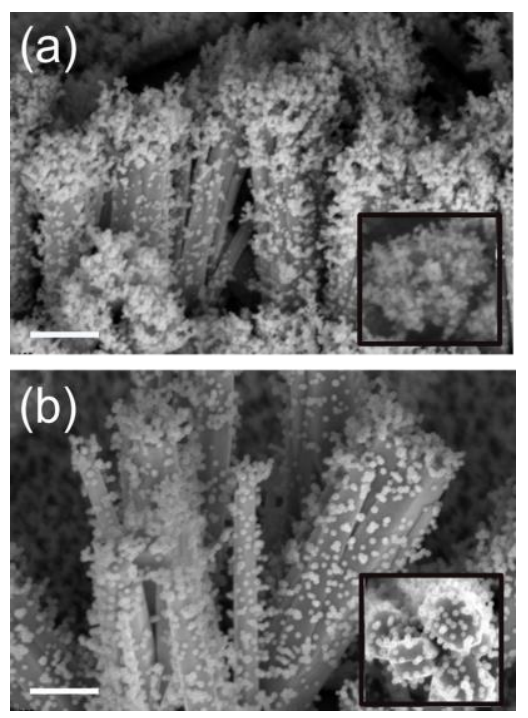

Figure 1: SEM images of the zinc oxide rods modified with copper nanoparticles with (a) high loading and (b) low loading of particles. The images were taken with a sample tilt of $54^{\circ}$ (main) and $0^{\circ}$ (inset). Scale bar indicates $400 \mathrm{~nm}$ in all cases. 
Cross sectional milling by FIB followed by SEM imaging successfully revealed the rods and the fibrillike aggregates, which are shown to consist of individual nanoparticles (typical diameter of $25 \mathrm{~nm}$ ) attached directly on top of each other to form structures which can extend $200 \mathrm{~nm}$ and $500 \mathrm{~nm}$ out from the surface in the case of low and high copper nanoparticle loading respectively (Figure 2). These types of structures are surprising since if the nanoparticles were falling randomly on the surface, a uniform coverage on the surfaces exposed to nanoparticle bombardment would be expected.
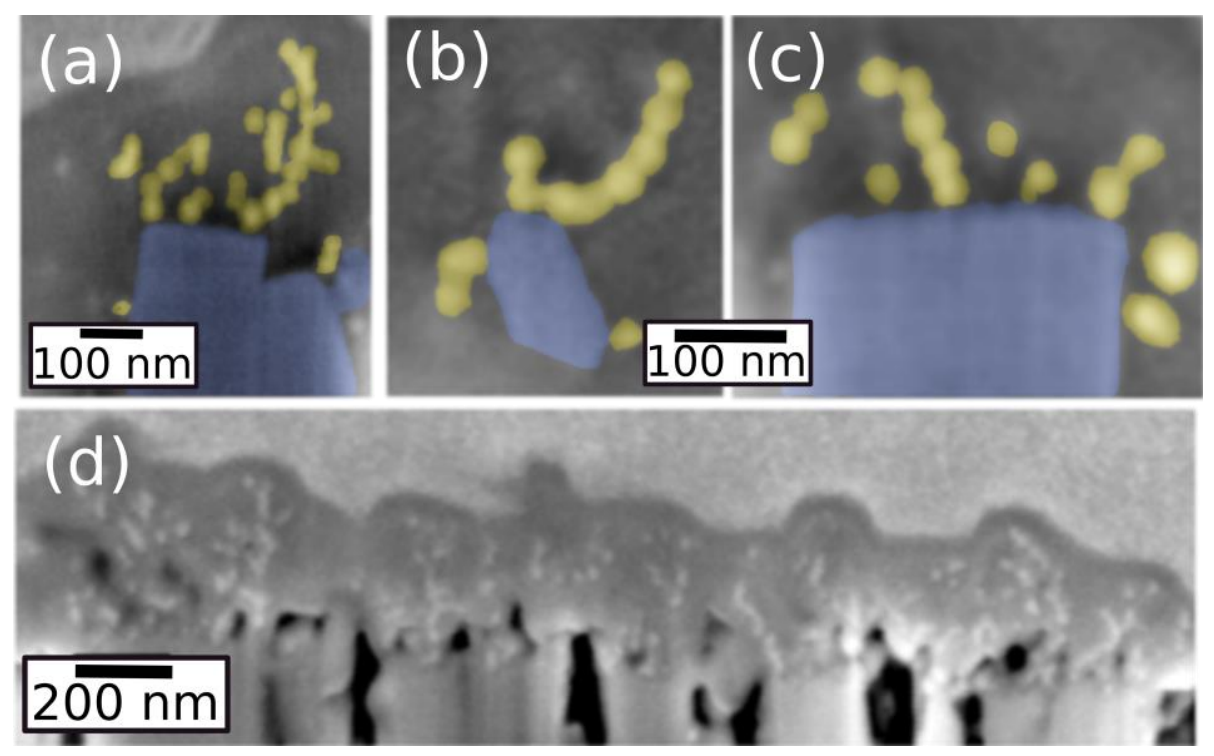

Figure 2: Cross sections produced by focused ion beam milling and imaged using scanning electron microscopy. (a) to (c) are artificially coloured SEM images taken from the sample with a low loading of copper nanoparticles (yellow) onto the zinc oxide rods (blue), which are revealed to be aggregated together to form fibrils which extend up to $200 \mathrm{~nm}$ from the rod surface (the original image is given in the supporting information). The protective platinum layer surrounds the particles, with the electron beam deposited material appearing darker compared to the ion beam. (d) is from the high loading of nanoparticle sample,fibrils of particles are seen extending up to $500 \mathrm{~nm}$.

Characterisation of interfaces between nanoparticles and nanoparticle to nanorod should reveal further the nature of these fibril-type structures. The results of the TEM analysis are summarized in Figure 3, which is complicated by the presence of 1- $2 \mathrm{~nm}$ sized crystals of platinum arising from the sample preparation procedure. Low resolution images clearly reveal the rods and copper nanoparticles forming the fibril-like features with EDS analysis confirming that the features seen in the FIB cross sections do, as expected, comprise of copper nanoparticles. High resolution imaging between both particles and particle to rod appear to show the respective crystal domains (facets) adhering directly to each other with no evidence of an interface layer. Such a layer may form due to the nature of the particles (such as an oxide layer) or an external source (such as a layer of adsorbents). 


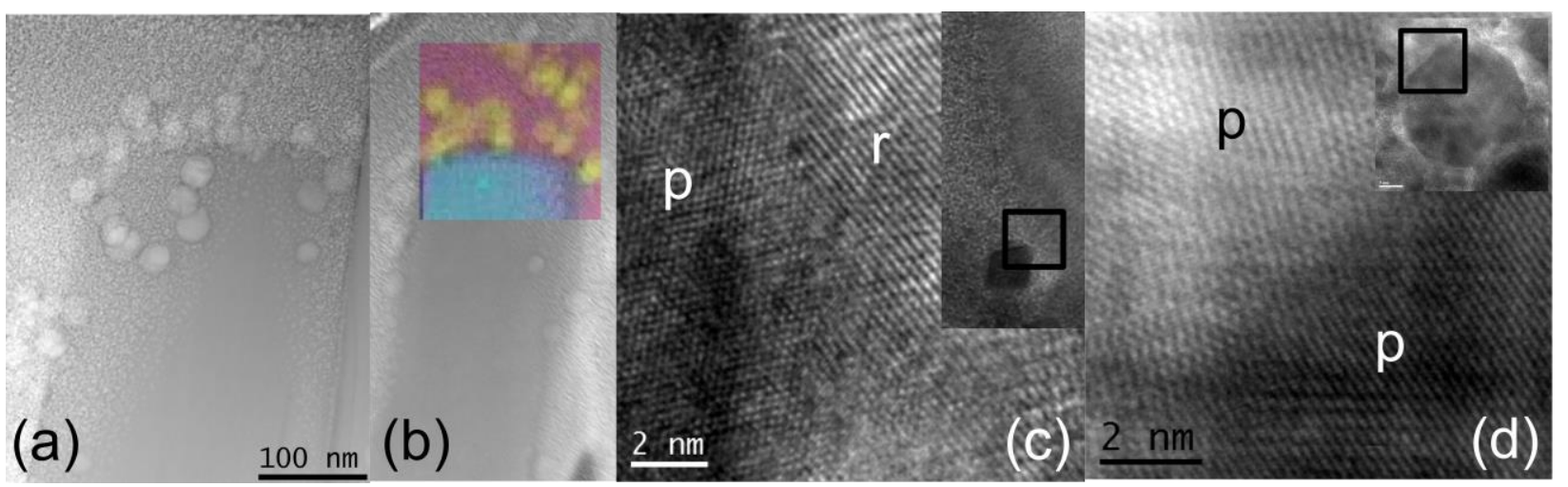

Figure 3: (S)TEM analysis of the rods and particles. (a) and (b) HAADF-STEM images revealing the zinc oxide rods and aggregated nanoparticles. In (b) a superimposed EDS map of the same area showing the dispersion of different materials is shown; zinc (blue), copper (yellow) and titanium (red). (c) and (d) bright field (inset) and high resolution TEM images of the interface between (c) a particle (p) and rod (r) and (d) two particles (square indicate the HRTEM image area.)

\section{Discussion}

The type of structures observed here cannot be derived by non-interacting particles falling and coating the rods. The structures suggest strong particle-particle affinity leading to particle aggregation (rather than agglomeration). This is supported by the TEM analysis showing direct attachment between the crystalline faces of the particles and rods. Diffusion limited aggregation (DLA) is often used to model aggregate structures and has been applied to nanoparticle aggregation in the past[13,14]. A custom written MATLAB code was used to simulate the nanoparticles structures formed by DLA by employing a random walk in three dimensions to simulate the particle movements. Rod like structures were defined at the bottom of a three dimensional matrix and then particles we added at the top. From the FIB and TEM analysis adhesion between two particles appears only to occur when their crystalline facet faces are in direct contact. Due to faceted nature of the nanoparticles only a limited number of possible adhesion points on the particle was allowed. However the model structures produced using this approach extend further from the rods than expected (Figure 4(a)). In order to resolve this the model was modified. Instead of completely free movement of the nanoparticles their vertical motion was set so they had a constant downward velocity. The rationale behind this arises both from bombardment from the argon gas and acceleration by and through the anode ring of the negatively charged particles towards the substrate. This produces structures which more closely resemble those observed, for example the SEM image of the high loading case (Figure 4(b,c)) and the FIB produced cross sections for the low loading case (Figure 4(d)) 
(a)

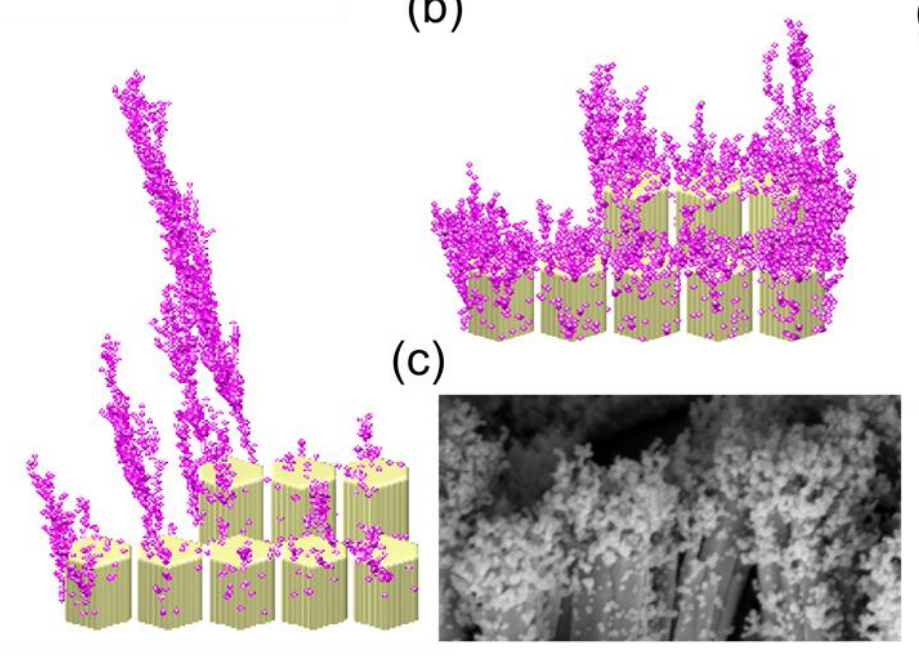

(d)

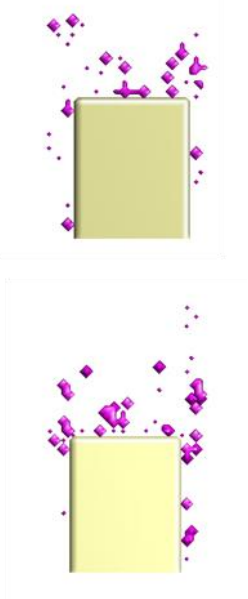

Figure 4: Simulated structures (a) produced using the DLA approach, (b) produced using the modified model where the rate of vertical movement is defined, (c) corresponding SEM image and (d) slice representations of single rods corresponding to the FIB cross section seen in Figure 2 (a,b).

Since the nanoparticles are generated in a plasma process, they attain a negative charge $[9,10]$. In case that the negatively charged nanoparticles fall onto the surface of the semiconducting $\mathrm{ZnO}$ rods and not losing their charge, following nanoparticles would expected to be repelled. This is apparently not the case as fibril-like structures of nanoparticles are built. A possible reason is that the charge is quickly distributed along the semiconducting surface, in turn forming a dipole moment and inducing a positive charge on the surface of the nanoparticles. This increases the probability that following nanoparticles attach to the deposited nanoparticles rather than to the surface of rods.

\section{Conclusions}

Here, anisotropic fibrils of copper nanoparticles of up to $500 \mathrm{~nm}$ in length formed on zinc oxide rods via the use of a pulsed plasma, are presented. These structures should display significantly different properties compared to a core shell structure formed due to a simple coating of nanoparticles. As such they hold great potential on forming the basis of news and improved products. Including those with improve catalytic properties.

\section{Acknowledgements}

This work was financially supported by the Knut and Alice Wallenberg Foundation under contract KAW 2014.0276 and the Swedish Research Council under grant 2008-6572 through the Linköping Linneaus Environment LiLi-NFM. 


\section{References}

[1] P.Y. Kim, J.-W. Oh, J.-M. Nam, Controlled Co-Assembly of Nanoparticles and Polymer into Ultralong and Continuous One-Dimensional Nanochains, J. Am. Chem. Soc. 137 (2015) 8030-8033. doi:10.1021/jacs.5b04714.

[2] Z. Tang, N.A. Kotov, One-Dimensional Assemblies of Nanoparticles: Preparation, Properties, and Promise, Adv. Mater. 17 (2005) 951-962. doi:10.1002/adma.200401593.

[3] X. Shen, L. Chen, D. Li, L. Zhu, H. Wang, C. Liu, Y. Wang, Q. Xiong, H. Chen, Assembly of Colloidal Nanoparticles Directed by the Microstructures of Polycrystalline Ice, ACS Nano. 5 (2011) 8426-8433. doi:10.1021/nn203399z.

[4] Y. Zhang, N. Zheng, K. Wang, S. Zhang, J. Wu, Effect of Copper Nanoparticles Dispersion on Catalytic Performance of $\mathrm{Cu} / \mathrm{SiO}_{2}$ Catalyst for Hydrogenation of Dimethyl Oxalate to Ethylene Glycol, J. Nanomater. 2013 (2013) e629375. doi:10.1155/2013/629375.

[5] J. Agrell, H. Birgersson, M. Boutonnet, I. Melián-Cabrera, R.M. Navarro, J.L.G. Fierro, Production of hydrogen from methanol over $\mathrm{Cu} / \mathrm{ZnO}$ catalysts promoted by $\mathrm{ZrO} 2$ and $\mathrm{Al2O} 3$, J. Catal. 219 (2003) 389403. doi:10.1016/S0021-9517(03)00221-5.

[6] R. Becker, H. Parala, F. Hipler, O.P. Tkachenko, K.V. Klementiev, W. Grünert, H. Wilmer, O. Hinrichsen, M. Muhler, A. Birkner, C. Wöll, S. Schäfer, R.A. Fischer, MOCVD-Loading of Mesoporous Siliceous Matrices with $\mathrm{Cu} / \mathrm{ZnO}$ : Supported Catalysts for Methanol Synthesis, Angew. Chem. Int. Ed. 43 (2004) 2839-2842. doi:10.1002/anie.200351166.

[7] I. Kasatkin, P. Kurr, B. Kniep, A. Trunschke, R. Schlögl, Role of Lattice Strain and Defects in Copper Particles on the Activity of $\mathrm{Cu} / \mathrm{ZnO} / \mathrm{Al} 2 \mathrm{O} 3$ Catalysts for Methanol Synthesis, Angew. Chem. Int. Ed. 46 (2007) 7324-7327. doi:10.1002/anie.200702600.

[8] L.-C. Wang, Y.-M. Liu, M. Chen, Y. Cao, H.-Y. He, G.-S. Wu, W.-L. Dai, K.-N. Fan, Production of hydrogen by steam reforming of methanol over $\mathrm{Cu} / \mathrm{ZnO}$ catalysts prepared via a practical soft reactive grinding route based on dry oxalate-precursor synthesis, J. Catal. 246 (2007) 193-204. doi:10.1016/j.jcat.2006.12.006.

[9] I. Pilch, D. Söderström, N. Brenning, U. Helmersson, Size-controlled growth of nanoparticles in a highly ionized pulsed plasma, Appl. Phys. Lett. 102 (2013) 33108-33108-4. doi:doi:10.1063/1.4788739. 
[10] I. Pilch, D. Söderström, M.I. Hasan, U. Helmersson, N. Brenning, Fast growth of nanoparticles in a hollow cathode plasma through orbit motion limited ion collection, Appl. Phys. Lett. 103 (2013) 193108. doi:10.1063/1.4828883.

[11] Y. khan, S.T. Hussain, M.A. Abbasi, P.-O. Kall, F. Söderlind, On the decoration of 3D nickel foam with single crystal ZnO nanorod arrays and their cathodoluminescence study, Mater. Lett. 90 (2013) 126-130. doi:10.1016/j.matlet.2012.09.033.

[12] R.M. Langford, A.K. Petford-Long, Preparation of transmission electron microscopy cross-section specimens using focused ion beam milling, J. Vac. Sci. Technol. Vac. Surf. Films. 19 (2001) 2186-2193. doi:10.1116/1.1378072.

[13] J. Tang, Z. Li, Q. Xia, R.S. Williams, Fractal Structure Formation from Ag Nanoparticle Films on Insulating Substrates, Langmuir. 25 (2009) 7222-7225. doi:10.1021/la9010532.

[14] N.H. Mack, J.A. Bailey, S.K. Doorn, C.-A. Chen, H.-M. Gau, P. Xu, D.J. Williams, E.A. Akhadov, H.-L. Wang, Mechanistic Study of Silver Nanoparticle Formation on Conducting Polymer Surfaces, Langmuir. 27 (2011) 4979-4985. doi:10.1021/la103644j. 